\title{
Experimental demonstration of an optical differentiator based on a fiber Bragg grating in transmission
}

\author{
Miguel A. Preciado,* Xuewen Shu, Paul Harper, and Kate Sugden \\ Aston Institute of Photonic Technologies, Aston University, Birmingham, B4 7ET, UK \\ *Corresponding author: m.preciado@aston.ac.uk
}

Received December 13, 2012; revised February 8, 2013; accepted February 11, 2013;

posted February 11, 2013 (Doc. ID 181696); published March 13, 2013

\begin{abstract}
We report the first experimental demonstration of single transmissive fiber Bragg grating implementation of a first-order optical differentiation. The device has been designed and fabricated, and the experimental results show a good performance over an operational bandwidth of $\sim 2 \mathrm{~nm}$. (c) 2013 Optical Society of America

OCIS codes: $\quad 060.3735,200.4740,230.1150,320.5540,320.7080$.
\end{abstract}

Optical differentiators were first proposed by Ngo and Binh [1], and constitute a basic device to analogue alloptical signal processing [2-5]. They perform a temporal differentiation of the complex field envelop (both amplitude and phase) of an arbitrary optical input signal at operation speeds several orders of magnitude higher than is possible using electronics. Many different schemes have been previously proposed [6-13]. Overall, fiber grating approaches [8-13], both fiber Bragg grating (FBG) and long period grating (LPG), are simple all-fiber approaches with interesting advantages, such as low cost, low insertion losses, and full compatibility with fiber optic systems. Although the LPG approach proposed in [9] have been proved to have a good performance in a regime of huge bandwidths (up to $19 \mathrm{~nm}$ ), FBGs may be preferred in applications with a bandwidth up to a few $\mathrm{nm}$, because of the extreme sensitivity of LPGs to environmental fluctuations [9]. However, the FBG approaches proposed in [9-12] inevitably require one or more additional optical elements, such as an optical circulator, coupler, or additional fiber grating to obtain a first-order differentiator.

An extremely simple, single optical-element FBG approach was proposed in [13] for first-order differentiation. It is well-known that the amplitude and phase of an FBG in transmission are related by the logarithmic Hilbert transform relation [14]. Using this relationship in the design process, it was theoretically and numerically demonstrated that a single FBG in transmission can be designed to simultaneously approach the amplitude and phase of a first-order differentiator spectral response, without the need for any additional elements.

In this Letter, we design, numerically simulate, and fabricate a first-order optical differentiator based on an FBG in transmission, using the ideas introduced in [13]. To prove the concept, we characterized the FBG with an optical vector analyzer, and performed an experiment of optical pulse differentiation where the signals were characterized using an optical spectrum analyser (OSA) and a second harmonic generation (SHG) frequency resolved optical gating (FROG) system [15].

The spectral response of the ideal first-order differentiator is $H_{\text {diff }}(\omega)=F_{\text {out }}(\omega) / F_{\text {in }}(\omega)=j \omega$, where $\omega$ is the base-band angular pulsation, i.e., $\omega=\omega_{\text {opt }}-\omega_{0}, \omega_{\text {opt }}$ is the optical angular pulsation, $\omega_{0}$ is the central angular frequency of the signals, and $j$ is the imaginary unit. This spectral response presents a $\pi$-phase shift at $\omega=0$.
As it was demonstrated in [13], an FBG in transmission can simultaneously obtain the amplitude and phase spectral response of a differentiator, since they are logarithm Hilbert transform (LHT) pairs, $\arg \left(H_{\text {diff }}(\omega)\right)=$ $\operatorname{LHT}\left(\left|H_{\text {diff }}(\omega)\right|\right)$. Therefore, the objective is to generate an FBG where the spectral response amplitude in transmission $\left|H_{T}(\omega)\right|$ approaches $\left|H_{\text {diff }}(\omega)\right|$ over a given bandwidth, automatically obtaining the corresponding spectral phase in transmission $\arg \left(H_{T}(\omega)\right)=$ $\operatorname{LHT}\left(\left|H_{T}(\omega)\right|\right)$.

Several aspects must be taken into account to generate a feasible design. An ideal differentiator spectral response would require a zero in transmission $(100 \%$ of reflectivity) at the central wavelength. We can obtain a good approximation by defining a limited operational bandwidth and a maximum transmission dip in the central wavelength. Also, a limited bandwidth of operation must be defined. Moreover, while the spectral response amplitude in reflection is uniquely defined by $\left|H_{R}(\omega)\right|=\left(1-\left|H_{T}(\omega)\right|^{2}\right)^{1 / 2}$, the spectral response phase in reflection $\arg \left(H_{R}(\omega)\right)$ is a degree of freedom in the design, and can be arbitrarily selected. However, it is very important to carefully select $\arg \left(H_{R}(\omega)\right)$ to obtain feasible FBG design parameters.

We designed and fabricated a first-order optical differentiator based on an FBG in transmission. The central zero in transmission is approached by a central transmission dip of $-40 \mathrm{~dB}$. We limited the bandwidth by windowing $\left|H_{R}(\omega)\right|$ with a $4 \mathrm{~nm}$ (total bandwidth) raised cosine function with a 0.1 roll-off factor. The spectral response phase in reflection, which is a degree of freedom in the design, has been defined as $\arg \left(H_{R}(\omega)\right)=$ $\arg \left(H_{T}(\omega)\right)+(T \omega)^{2}$, with $T=11.756 \mathrm{~ns}$, where $T$ is a design constant, used to define the quadratic phase term in $\arg \left(H_{R}(\omega)\right)$. All these specifications have been carefully selected to obtain a design that is feasible to fabricate with our system, resulting in a $9.8 \mathrm{~cm}$ FBG with the coupling coefficient and grating period (calculated by inverse scattering [16]) shown in Fig. 1. The designed grating structure was fabricated with the UV laser direct-writing system developed at Aston University, which allows the grating to be created pitch-by-pitch. The coupling coefficient profile and the varied period were realized by appropriately controlling the ON/OFF of an acoustic optical modulator and moving the phase mask/fiber. The grating structure was made in hydrogen-loaded photosensitive fiber and then stabilized 

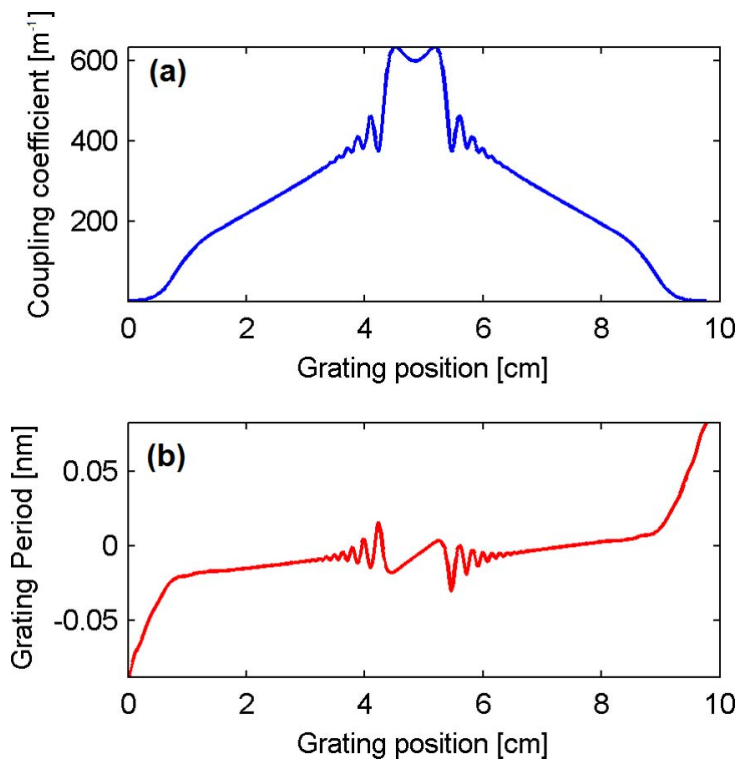

Fig. 1. (Color online) (a) Coupling coefficient and (b) grating period of the designed FBG obtained from inverse scattering.

by

annealing at $80^{\circ} \mathrm{C}$ for $60 \mathrm{~h}$ after the fabrication. The fabricated FBG was characterized using an optical vector analyzer, obtaining the amplitude and phase of the spectral response in transmission, which is shown in Fig. 2, with the simulation results that were generated using the exact value of the designed grating coupling coefficient $\kappa(z)$, and also $0.9 \cdot \kappa(z)$, and $0.8 \cdot \kappa(z)$. The central transmission dip obtained is $\sim-30 \mathrm{~dB}$ instead of $-40 \mathrm{~dB}$, and some spectral ripples can be observed due to fabrication process errors. It is worth noting that the cladding losses have a very marginal effect in the operational band of the differentiator. It is particularly interesting to observe the required spectral $\pi$-phase shift, as theoretically predicted in [13], showing that both am-
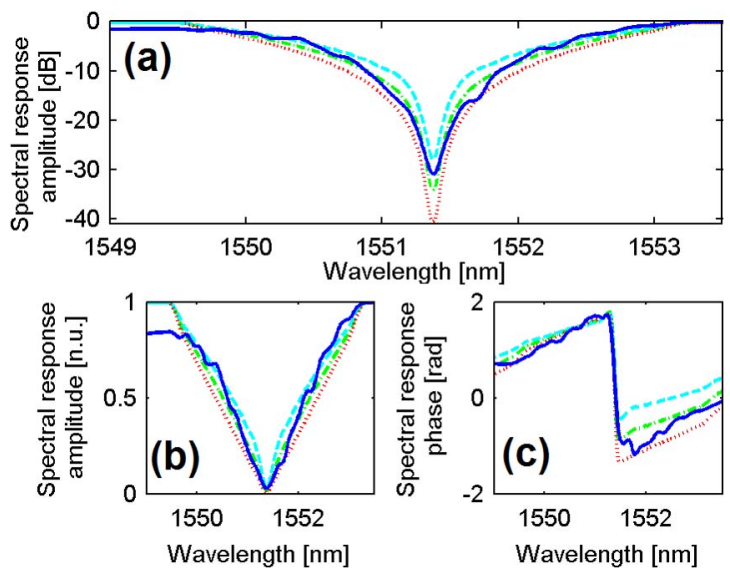

Fig. 2. (Color online) Comparison between the spectral response in transmission of the experimentally characterized FBG (solid blue), and the simulation results using the designed coupling coefficient $\kappa(z)$ (dotted red), and the coupling coefficient with a $10 \%$ variation $0.9 \cdot \kappa(z)$ (dash-dotted green), and a $20 \%$ variation $0.8 \cdot \kappa(z)$ (dashed cyan).

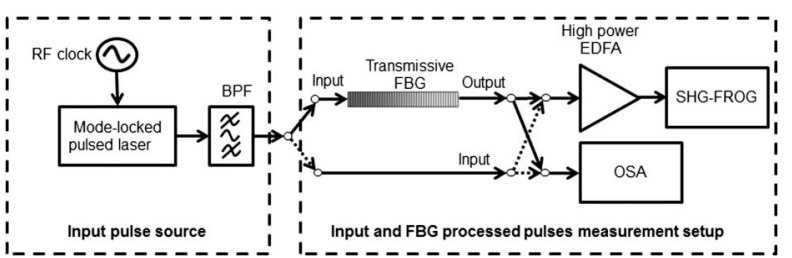

Fig. 3. Experimental setup used to prove the operation of the fabricated FBG as a differentiator. BPF, bandpass filter; EDFA amplifier.

plitude and phase differentiator response can simultaneously be obtained by using a single device.

To prove the operation of the fabricated device, the experimental setup shown in Fig. 3 was used. The input pulses were generated by a $10 \mathrm{GHz}$ active mode-locked pulsed fiber laser (Pritel Inc.). The bandwidth (and temporal width) was adjusted using an optical bandpass filter, where a bandwidth of approximately $2 \mathrm{~nm}$ at $10 \mathrm{~dB}$ was selected. The resulting input pulses and processed output pulses were then characterized using an SHG-FROG system and an OSA. Figure $\underline{4}$ shows the SHG-FROG traces of the input and output pulses from the FROG recovery algorithm. The FROG recovered spectrum, shown in Fig. 5, has a good agreement with the input spectrum directly measured with the OSA. However, there is a slight mismatch at longer wavelengths that would have an undesirable effect on the calculated differentiated pulse, since these spectral components are magnified. To avoid this effect, the FROG recovered spectrum was filtered by applying a 10-order (a)

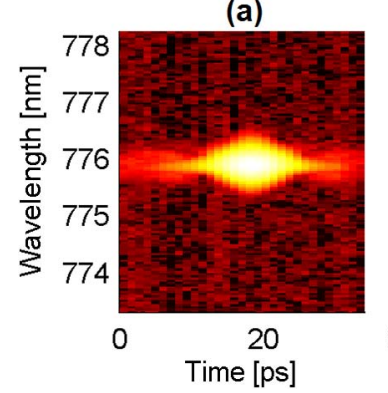

(b)

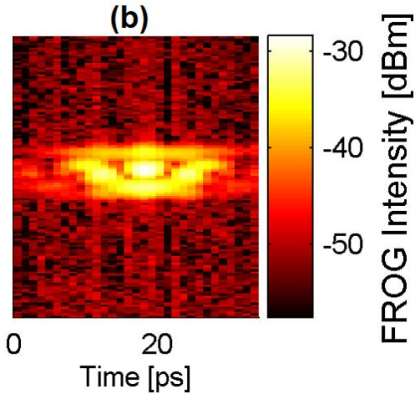

Fig. 4. (Color online) (a) SHG-FROG traces for the input pulse and (b) differentiated output pulse.

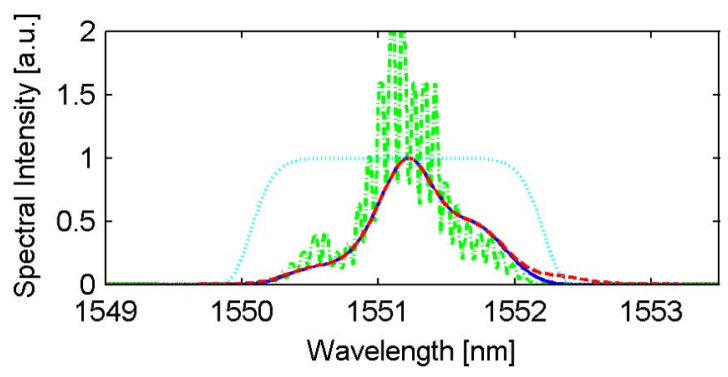

Fig. 5. (Color online) OSA (dash-dotted green), FROG (dashed red), corrected windowed FROG (solid blue) input pulse spectral intensity, and 10-order super-Gaussian window (dotted cyan). 


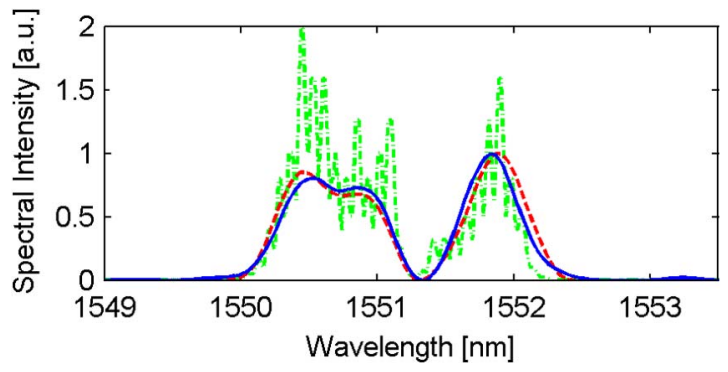

Fig. 6. (Color online) OSA (dash-dotted green), FROG trace (solid blue), and numerically calculated (dashed red) output pulse spectral intensity.
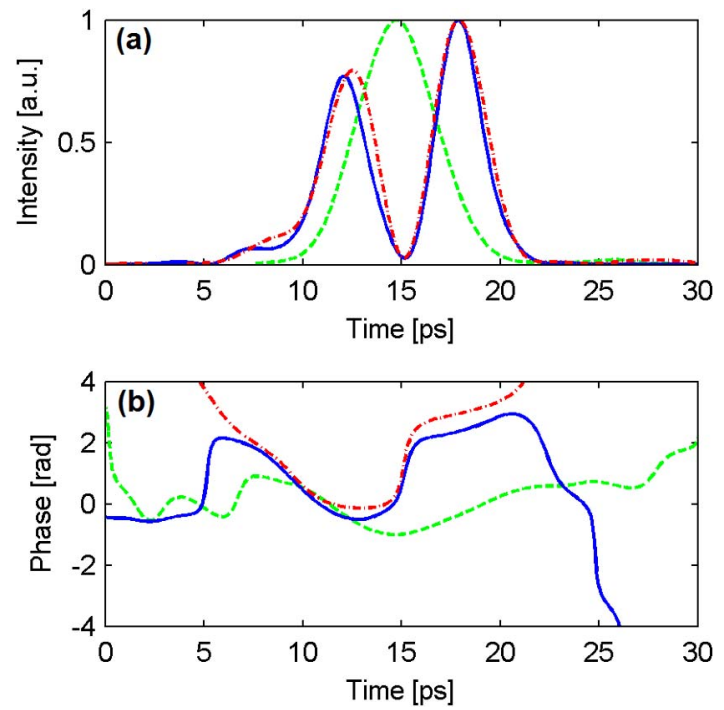

Fig. 7. (Color online) (a) FROG recovered temporal intensity and (b) phase of the input pulse (dashed green), ouput pulse (solid blue), and numerically calculated temporal differentiation (dash-dotted red).

super-Gaussian spectral window, obtaining a spectrum in better agreement with the OSA spectrum (see Fig. 5).

Regarding the output signal, the FROG recovered output spectrum is shown in Fig. $\underline{6}$, compared to the directly OSA measured output spectrum and the numerically calculated differentiated input pulse. Finally, Fig. 7 shows the temporal intensity and phase of the input pulse, output pulse, and the numerically calculated differentiated input pulse, showing a good agreement in temporal intensity and phase, and proving the validity of the operation of the fabricated differentiator. It is worth noting that the asymmetry of the results in both the temporal and spectral domain is due to the asymmetry of the spectrum of the input (Fig. 5). However we can observe a good agreement in temporal intensity and phase from both FBG and ideal first-order differentiator, proving the validity of the operation of the fabricated differentiator, with a processing accuracy of $99.38 \%$, and an energy efficiency of $6.2 \%$. The processing accuracy has been es- timated with the normalized cross-correlation coefficient corr $=\int I_{1}(t) I_{2}(t) \mathrm{d} t / \sqrt{\int I_{1}^{2}(t) \mathrm{d} t \int I_{2}^{2}(t) \mathrm{d} t}$, where $I_{1}(t)$ is the experiment output intensity, and $I_{2}(t)$ is the numerically calculated derivative. The energy efficiency has been estimated as the ratio of the output pulse energy over the input pulse energy.

In summary, in this Letter we have performed the first experimental demonstration of a first-order temporal differentiator based on a single FBG in transmission without the need of any additional optical elements. As it was theoretically demonstrated in [13], the logarithmic Hilbert transform relationship between the amplitude and phase of an FBG in transmission spectral response results in a required $\pi$-phase shift in the phase when the corresponding amplitude approximates the first-order differentiator spectral response amplitude.

We have proven that the resulting differentiated pulse is in very good agreement with the numerically predicted differentiated pulse. However, we could also observe that the fabrication errors result in some ripples and mismatch in the resulting differentiator FBG spectral response, introducing some spectral distortion in the output signal. However, this does not notably affect the resulting differentiated pulse temporal intensity. In conclusion, this experimentally demonstrated how single FBG approach constitutes a very simple and inexpensive solution, appearing to be a very attractive alternative for the implementation of first-order optical differentiators.

This research was supported by a Marie Curie Intra European Fellowship within the 7th European Community Framework Programme.

\section{References}

1. N. Q. Ngo and L. N. Binh, Fiber Integr. Opt. 14, 359 (1995).

2. F. Li, Y. Park, and J. Azaña, Opt. Lett. 32, 3364 (2007).

3. A. Consoli, J. M. G. Tijero, and I. Esquivias, Opt. Express 19, 10805 (2011).

4. R. Slavik, Y. Park, and J. Azaña, Opt. Express 15, 6717 (2007).

5. J. Azaña, IEEE Photon. J. 2, 359 (2010).

6. N. Q. Ngo, S. F. Yu, S. C. Tjin, and C. H. Kam, Opt. Commun. 230, 115 (2004).

7. Y. Park, R. Slavik, and J. Azaña, Opt. Lett. 32, 710 (2007).

8. R. Slavík, Y. Park, M. Kulishov, R. Morandotti, and J. Azaña, Opt. Express 14, 10699 (2006).

9. N. K. Berger, B. Levit, B. Fischer, M. Kulishov, D. V. Plant, and J. Azaña, Opt. Express 15, 371 (2007).

10. L.-M. Rivas, K. Singh, A. Carballar, and J. Azaña, IEEE Photon. Technol. Lett. 19, 1209 (2007).

11. M. A. Preciado, V. Garcia-Muñoz, and M. A. Muriel, Opt. Express 15, 7196 (2007).

12. M. Li, D. Janner, J. Yao, and V. Pruneri, Opt. Express 17, 19798 (2009).

13. M. A. Preciado and M. A. Muriel, Opt. Lett. 33, 2458 (2008).

14. J. Skaar, J. Opt. Soc. Am. A 18, 557 (2001).

15. R. Trebino and D. J. Kane, J. Opt. Soc. Am. A 10, 1101 (1993).

16. R. Feced, M. N. Zervas, and M. A. Muriel, IEEE J. Quantum Electron. 35, 1105 (1999). 